\title{
Full-Field Displacement Measurement Technique by Using Repeated Patterns and JPEG Compressed Images
}

\author{
Shien Ri ${ }^{1,}$, , Satoshi Hayashi ${ }^{1,2}$, Hiroshi Tsuda ${ }^{1}$, Shinji Ogihara ${ }^{2}$ \\ ${ }^{1}$ Research Institute of Instrumentation Frontier, National Institute of Advanced Industrial Science and Technology, Tsukuba, Japan \\ ${ }^{2}$ Department of Mechanical Engineering, Tokyo University of Science, Chiba, Japan
}

Email address:

ri-shien@aist.go.jp (S. Ri)

\section{To cite this article:}

Shien Ri, Satoshi Hayashi, Hiroshi Tsuda, Shinji Ogihara. Full-Field Displacement Measurement Technique by Using Repeated Patterns and JPEG Compressed Images. Optics. Special Issue: Optical Techniques for Deformation, Structure and Shape Evaluation.

Vol. 4, No. 3-1, 2015, pp. 9-13. doi: 10.11648/j.optics.s.2015040301.13

\begin{abstract}
This paper presents an experimental technique for measuring displacement distribution from an image of repeated patterns and JPEG compressed images. The measurement accuracy of the developed method is insusceptible to the degree of JPEG compression because only lower spatial frequency components of the repeated pattern are used to calculate the displacement distribution, and high spatial frequency components have high priority to cut-off according to the JPEG compression algorithm. The insensitivity to the JPEG compression was confirmed by a displacement measurement using JPEG images of various compression ratios or image qualities. We found that by using the highest quality JPEG image, the accuracy of displacement measurement could research 1/1000 of the repeated pattern pitch, as same the uncompressed BMP images. In addition, displacement with accuracy of 1/500 pitch could be measured from a compressed JPEG image of which the file size was $1 / 50$ of that of the BMP image. This technique is useful for measurement using a high-speed camera with high-resolution digital images for a long-term or wireless image transfer.
\end{abstract}

Keywords: Deformation Measurement, Sampling Moiré Method, Repeated Pattern, Compressed image, Phase Analysis

\section{Introduction}

Displacement distribution gives useful information to understand the deformation behavior in materials science and to ensure health monitoring of infrastructures such as bridges and buildings. Both mechanical contact displacement sensor and noncontact laser Doppler vibrometer (LDV) [1] have widely used in displacement measurement. These instruments, however, are basically point measurement systems and are quite costly for distribution analysis.

Compared with point measurement method, image-based measurement using a digital camera attracts a lot of attention because its simple setup, low cost, high accuracy and full-field measurement $[2,3]$. The image-based methods can be classified in terms of the patterns of the target marker. Digital image correlation (DIC) [4-6], as well known, uses random patterns in a subset region to determine the displacement distribution by using the images before and after deformations. On the other hand, various moiré methods [7-9] use regular and periodical patterns as the marker to measure displacement, strain and residual stress.
We recently developed a fast, simple, and accurate small-displacement distribution measurement technique [10] using arbitrary repeated patterns based on the sampling moiré method [11]. By analyzing digital images of a regular repeated patterns with known pitch on the materials or structures, we can obtain in-plane displacement distribution across the target $[12,13]$. To improve the measurement accuracy for arbitrary repeated patterns, in this method, we use not only the first frequency component, but also use the multiple low frequency components of the moiré fringes.

To perform accurate displacement distribution measurement, generally, RAW or bitmap (BMP) images of lossless compression are used in most image-based measurement methods. Recent digital cameras feature high resolution and fast recording. For example, using the Nikon digital single-lens reflex (DSLR) camera D800/D800E, we can capture a high-resolution image with 7360 by 4912 pixels in speed of 4 frames per second. This means if the image format is chose as RAW (lossless compression; 14-bit record), the size of each image is $41.3 \mathrm{MB}$. In case that we capture continually for 10 seconds, the image size is bigger than $1 \mathrm{~GB}$. On the other hand, if the image format is chosen 
as the JPEG (basic quality) compressed format, the image size can be reduced to $4 \mathrm{MB}$, i.e., 10 times smaller compared with the RAW image. By the way, image compression is achieved by removing the high spatial frequency components in the JPEG algorithm [14]. Therefore, we consider that our developed image-based method is insusceptible to the influence of JPEG compression because only low spatial frequency components are used.

In this study, an experimental technique for measuring displacement distribution is proposed, by using a combination of repeated patterns and JPEG compressed images. The effectiveness is confirmed by a displacement measurement experiment by using JPEG images for various qualities. This technique could be useful for measurement using a high-speed camera with high-resolution digital images for a long-term experiment to analyze a great number of images.

\section{Principle}

\subsection{Displacement Measurement Using Repeated Patterns}

The principle of displacement distribution measurement by using repeated patterns is presented in Fig. 1. By capturing an image of a periodic pattern with a pitch of $P$ in the image plane, the intensity of the pattern with an initial phase $\varphi_{0}$ can be presented in Eq. (1), as shown in Fig. 1(a).

$$
I(x, y)=\sum_{w=1}^{W} I_{a}(x, y ; w) \cos w\left\{2 \pi \frac{x}{P}+\varphi_{0}\right\}+I_{b}(x, y)
$$

where $I_{a}$ and $I_{b}$ present the amplitudes of the grating intensity with $w$-order frequency, and the background intensity, respectively. After performing down-sampling and intensity-interpolation, the multiple phase-shifted moiré fringe patterns can be obtained, as shown in Fig. 1(b), as expressed

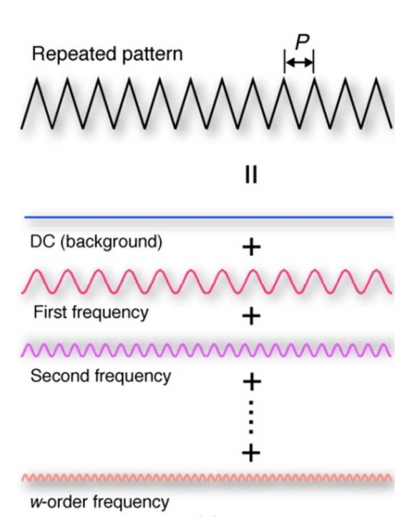

(a)

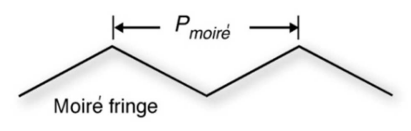

II

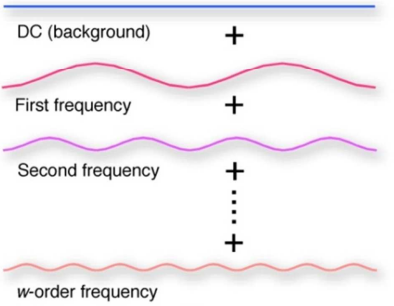

(b)

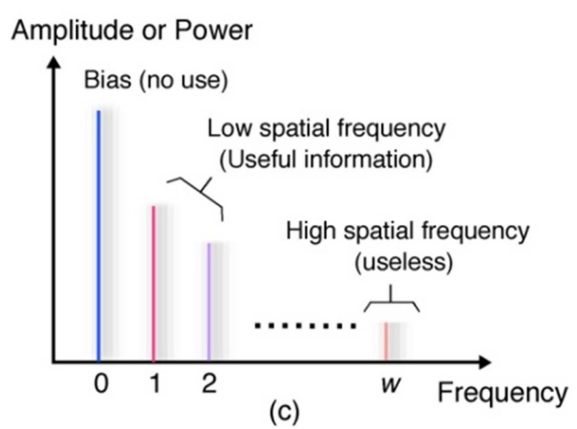

Figure 1. Displacement measurement by using a repeated pattern: (a) the arbitrary repeated pattern, (b) moire fringe obtained by the image processing of down-sampling and intensity interpolation, and (c) Fourier spectrum of the moiré fringe.

$$
\begin{gathered}
I_{m}(x, y)=\sum_{w=1}^{W} I_{a}(x, y ; w) \cos w\left\{2 \pi\left(\frac{1}{P}-\frac{1}{T}\right) x+\varphi_{0}+2 \pi \frac{k}{T}\right\}+I_{b}=\sum_{w=1}^{W} I_{a}(x, y ; w) \cos w\left\{\varphi_{m}(x, y)+2 \pi \frac{k}{T}\right\}+I_{b}, \\
(k=0,1, \cdots, T-1)
\end{gathered}
$$

The phase distribution of the moire fringe and the amplitude with frequency $w$ can be calculated by phase-shifting methods using discrete the Fourier transform (DFT) algorithm, as presented in Eq. (3) and Eq. (4), respectively.

$$
I_{a}(w)=\frac{2}{T} \sqrt{\left[\sum_{k=0}^{T-1} I_{m}(k) \cos w\left(2 \pi \frac{k}{T}\right)\right]^{2}+\left[\sum_{k=0}^{T-1} I_{m}(k) \sin w\left(2 \pi \frac{k}{T}\right)\right]^{2}}
$$

$$
\varphi_{m}(w)=-\arctan \frac{\sum_{k=0}^{T-1} I_{m}(k) \sin w\left(2 \pi \frac{k}{T}\right)}{\sum_{k=0}^{T-1} I_{m}(k) \cos w\left(2 \pi \frac{k}{T}\right)}
$$

The in-plane displacement distribution can be measured from the phase difference of the moiré fringe with a $w$-order frequency before and after deformations as

$$
\delta(x, y ; w)=-\frac{p}{2 \pi w} \Delta \varphi_{m}(x, y ; w)
$$

As shown in Fig. 1(c), the final displacement distribution, by considering multiple low spatial frequency components in the Fourier spectrum, can be obtained as follows

$$
\begin{gathered}
\hat{\delta}(x, y)=\sum_{w=1}^{W_{h}} \hat{a}(x, y ; w) \cdot \delta(x, y ; w) \\
\hat{a}(x, y ; w)=\frac{a(x, y ; w)}{\sum_{w=1}^{W_{h}} a(x, y ; k)}
\end{gathered}
$$

In our method, not only first frequency component but also multiple low spatial frequencies are used simultaneously to determine the in-plane displacement distribution. Therefore, 
this method allows accurate measurement using arbitrary repeated patterns on the structure surface. In the case of $\mathrm{w}=1$, it corresponds to the sampling moiré method [11].

\subsection{Outline and Algorithm of JPEG Compression}

As well known, the JPEG compression algorithm [14] is one of the digital image compression standards designed for compressing either full-color or gray images. In the JPEG algorithm, the digital image is compressed by discarding the high frequency components in the image because the low frequency components are more sensitive to human eye. JPEG compression is achieved in a series of steps, as shown in Fig. 2.
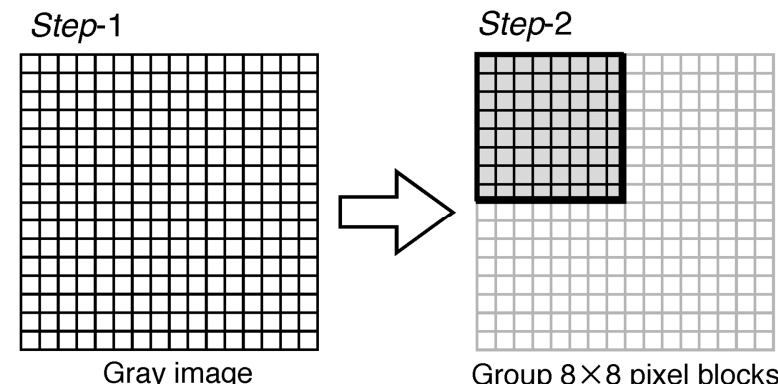

Group $8 \times 8$ pixel blocks
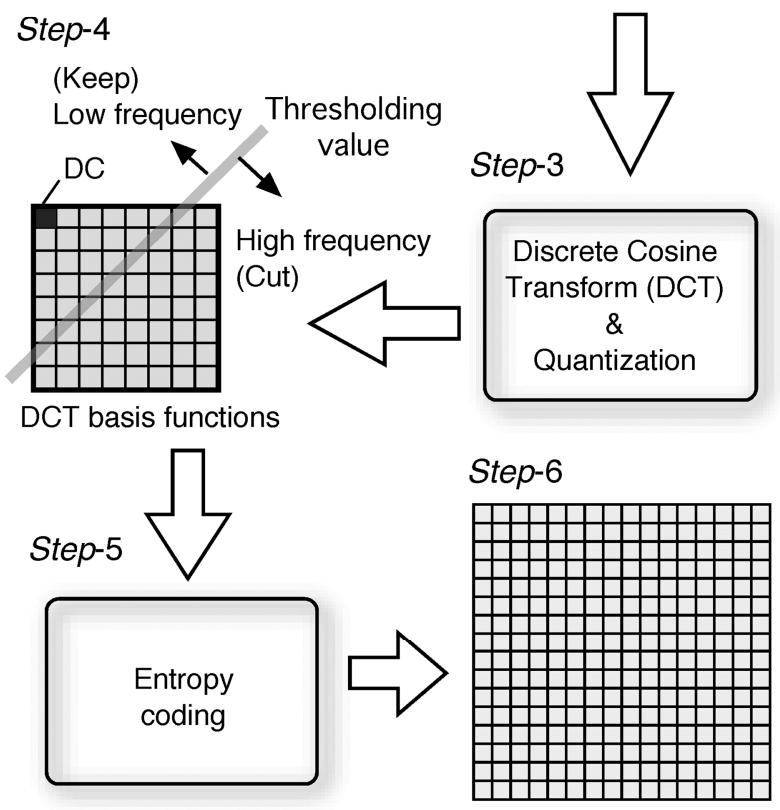

Step-6

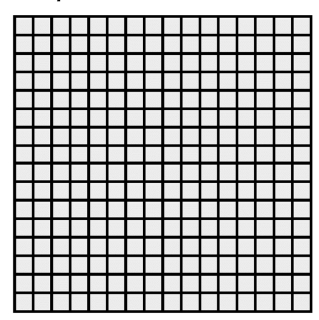

Compressed image

Figure 2. Image compression procedure by the JPEG algorithm.

Step-1) A RAW color or gray image with lossless compression is captured or loaded. In this study, we mainly focus on the gray image.

Step-2) The pixels of the whole image are grouped into blocks of 8 by 8 pixels for the RAW image.

Step-3) A discrete cosine transform (DCT) is applied to each block. The transform generates $1 \mathrm{DC}$ (background) and 63 AC (amplitude) components of each spatial frequency. Then, each of the 64 DCT components is quantized in conjunction with a quantization table. This is done in such a way that high spatial-frequency components are quantized with higher quantization coefficients than the lower one.

Step-4) After quantization, the results are rounded to an integer. As a consequence, these AC components almost become zero according to a thresholding value by choosing a compression ratio (i.e., image quality). This causes irretrievably lost of original detailed information.

Step-5) The 64 quantized frequency components are encoded by using a combination of the run length encoding and the Huffman coding.

Step-6) Finally, a compressed JPEG image with small size is generated and saved. The image size depends on the image quality factor (IQF).

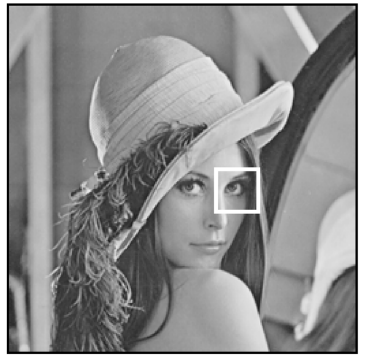

Original image

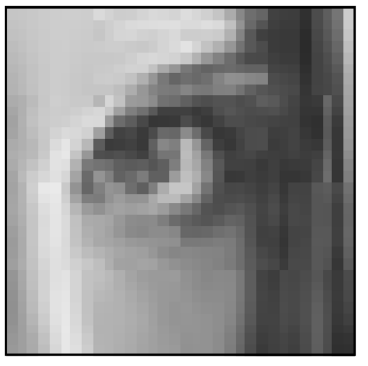

JPEG, IQF=50 (6.9 KB)
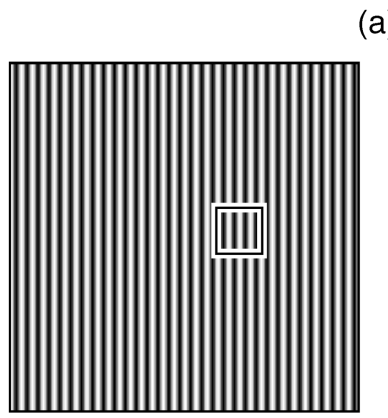

Original image

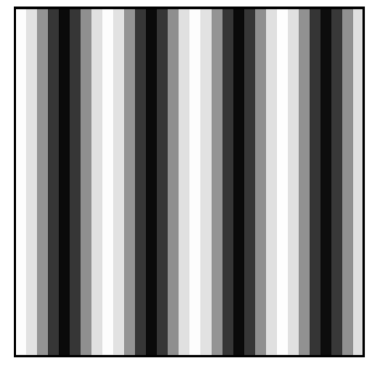

JPEG, IQF=50 (4.0 KB)

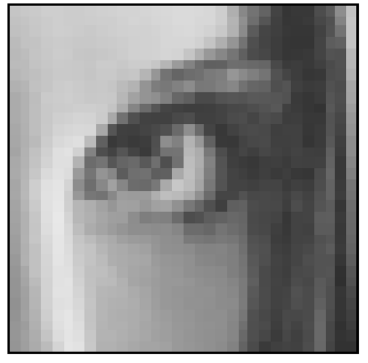

$\mathrm{BMP}(65.0 \mathrm{~KB})$

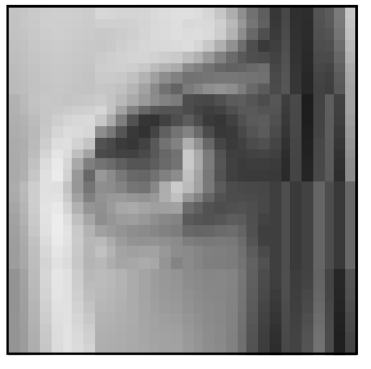

JPEG, IQF=20 (3.1 KB)
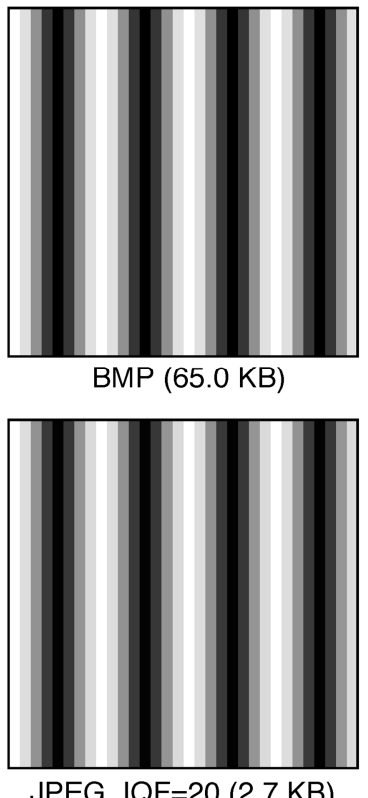

(b)
Figure 3. Comparison of (a) a general photograph and (b) a cosinusoidal grating under different image compression ratio or image quality factor. 
Figure 3 shows the compression results for a photograph of "Lena" and a repeated pattern of "cosinusoidal grating" with 256 by 256 pixels by using JPEG compression with different IQFs. In the JPEG image of "Lena", the block noise of 8 by 8 pixels becomes conspicuous with the decreasing IQF, as shown in the enlarged part (the image size is 32 by 32 pixels) in Fig. 3(a). On the other hand, such a block noise is hardly observed in the JPEG image of "cosinusoidal grating" with a low IQF, as shown in Fig. 3(b). The reason is that the cosinusoidal grating has only low spatial frequency component and high spatial frequency components have high priority to cut-off according to the JPEG compression algorithm.

\subsection{Combination of Repeated Patterns and JPEG Compressed Images}

From the results of Fig. 3, we found that our method as mentioned in Sec. 2.1 can be considered that the measurement accuracy is insusceptible to JPEG compression. In addition, the high frequency components due to camera noise can be cut off in the process of image compression. That is to say the combination of our displacement measurement method using repeated patterns and JPEG compressed images acts well for accurate displacement distribution measurement.

\section{Experiment}

\subsection{Experimental Setup}

Here, a simple displacement experiment was performed to investigate the relationships between displacement accuracy, the JPEG IQF, and the file size.

Figure 4 shows the experimental setup. A charge couple device (CCD) camera (The Imaging Source, DMK 41BF02.H; Germany) with 1280 vertical pixels and 960 horizontal pixels was used to capture the images. A flat plate with $10 \mathrm{~mm}$ repeated pitch of a rectangular, two alphanumeric characters, and a Chinese character was fixed on a linear moving stage (Suruga Seiki, KX1250C-R; 1 m resolution; Japan). These patterns were moved in the horizontal direction from $0 \mathrm{~mm}$ to $1.0 \mathrm{~mm}$ by $0.02 \mathrm{~mm}$ steps, and a single image without compression was captured and saved at each position. The distance between the $\mathrm{CCD}$ and the repeated pattern was 1.35 $\mathrm{m}$, and the focus length of the camera lens was $12 \mathrm{~mm}$. In this case, one pitch of each pattern corresponded to approximately 20 pixels in the captured image. Therefore, the down-sampling pitch was set to be 20 pixels in the displacement analysis, and a linear intensity interpolation was performed.

In the evaluation of measurement accuracy, the captured BMP images were converted to JPEG images with various compression ratio from IQF $=100$ (good quality) to IQF $=10$ (poor quality). For the rectangular pattern, the first frequency component was only used to analyze the displacement distribution; for the other repeated patterns including an alphanumeric characters and a Chinese character, the multiple frequency components from the first- to the fifth-order were used to analyze the displacement distribution in the analysis. The high frequency components having amplitude less than 3\% of that of the first frequency are not used in order to consider the random noise in an actual experiment, because random noise easily appears in high frequency with a low amplitude. The root mean square (RMS) error of 60 by 40 pixels in the central evaluation area at each position was evaluated.

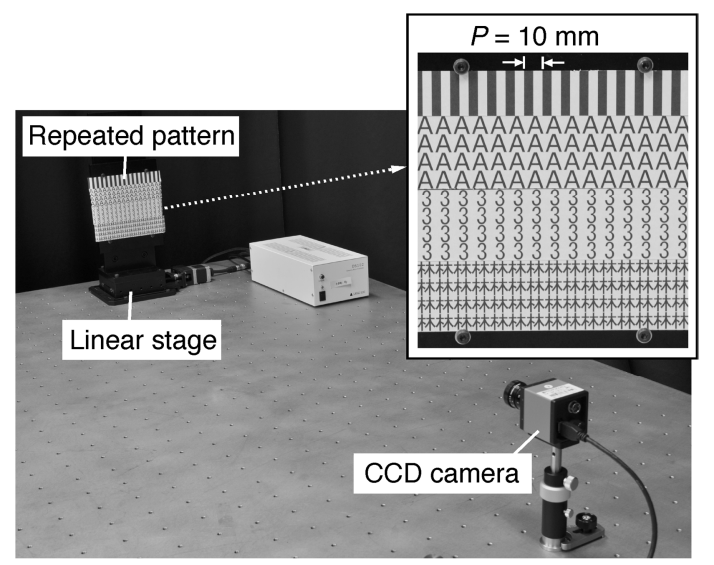

Figure 4. Experimental setup. A digital CCD camera and a repeated pattern with a pitch of $10 \mathrm{~mm}$ were used to investigate the displacement measurement accuracy for various image quality factor of JPEG.

\subsection{Experimental Results and Discussion}

Figure 5 shows the analyzed results of the displacement measurement RMS errors for three different repeated patterns using BMP and various JPEG IQFs. Here, only the results of JPEG IQF $=100,50$, and 20 show the effect of compression. For the rectangular pattern, as shown in Fig. 5(a), RMS error was approximately $0.1 \mathrm{~mm}$ in JPEG image quality factor from $I Q F=50$ to $I Q F=100$. In case of $I Q F=20, R M S$ error was approximately ranging from $0.01 \mathrm{~mm}$ to $0.02 \mathrm{~mm}$.

Figures 5(b) and 5(c) show the analyzed results of the displacement measurement RMS errors using the alphabetical character " $\mathrm{A}$ " and the Chinese character “林”, respectively. It is noteworthy in both cases that the JPEG image with IQF of 100 shows better accuracy than that of the BMP format image. These interesting results are attributable to the compression algorithm of the JPEG image. In the JPEG compression, the low frequency components containing useful information in the displacement measurement analysis remain. On the other hand, high spatial frequency components which correspond to image noise are eliminated by the JPEG compression. It is implied that high accurate displacement measurement can be performed with a small image size by using the JPEG algorithm with a proper IQF.

In the range of JPEG IQF $=90$ to $\mathrm{IQF}=10$, the RMS error increases with the decreasing JPEG IQF, as shown in Figs. 5(b) and 5(c). The relationship between the JPEG image quality from 10 to 100 and the image file size for the repeated pattern of “林” is shown in Fig. 6(a). In the case of $\mathrm{IQF}=90$, the image file size can be reduced by 10 times. In case of $\mathrm{IQF}=50$, the image file size can be reduced to 30 times compared with the BMP format. 


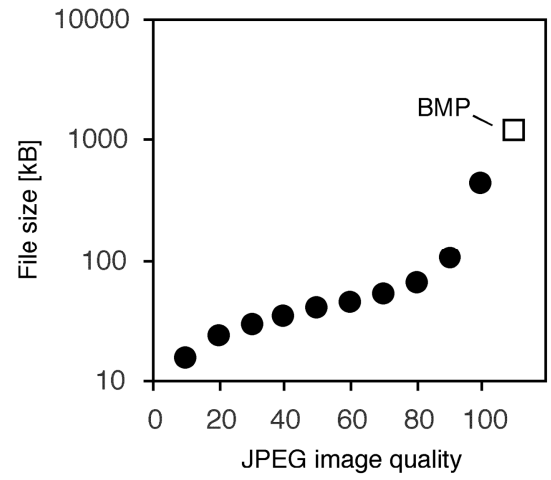

(a)

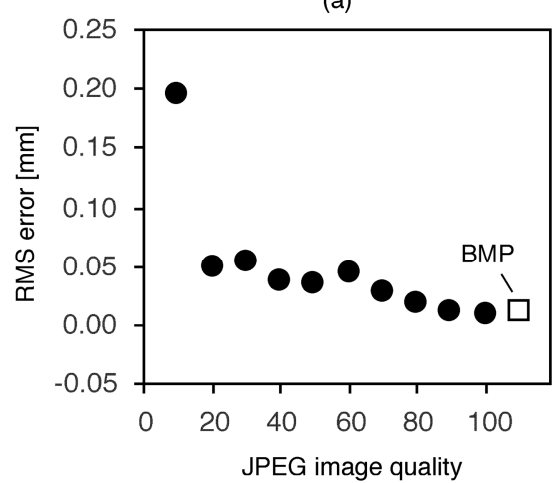

(b)

Figure 6. Relationships between the JPEG image quality, the file size and the RMS error of displacement measurement in the case of the repeated pattern of “林”: (a) JPEG image quality vs. file size, (b) JPEG image quality vs. RMS displacement error.

Figure 6(b) shows the relationship between the JPEG image quality and the RMS displacement error. Clearly, JPEG IQF=10 cannot be analyzed accurately because the RMS error dramatically increases. As well known, the image quality should not be lower than $\mathrm{IQF}=20$ for most photograph applications. In the case of $\mathrm{IQF}=20$, the measurement accuracy is $0.05 \mathrm{~mm}$, and it corresponds to $1 / 200$ pitch of the repeated pattern. For accurate measurement, a IQF higher than 50 should be chosen. Experimental results demonstrate that image compression with a proper IQF is useful to the sampling moiré method for measuring the accurate in-plane displacement distribution.

\section{Conclusions}

This paper presented an accurate displacement distribution measurement technique by using repeated patterns and JPEG compressed images with small image sizes. The measurement accuracy was quantitatively investigated by a displacement measurement experiment by comparing lossless compression BMP images and JPEG compressed images with various image qualities.

Experimental results indicated that a $1 / 1000$ pitch displacement could be accurately detected using a JPEG image in the case of the highest image quality (IQF=100). For the rectangular pattern, using JPEG IQF=20, an accuracy of $1 / 500$ pitch can be analyzed and the image file size is 50 times smaller than the BMP format. Therefore, this method could be useful for the purpose of measurement using a high-speed camera with high-resolution digital images for a long-term recording to reduce the extremely large image size.

\section{References}

[1] H. Nassif, M. Gindy, and J. Davis, "Comparison of laser Doppler vibrometer with contact sensors for monitoring bridge deflection and vibration," NDT \& E International, 2005, pp. 213-218.

[2] A. M. Wahbeh, J. Caffrey, and S. Masri, "A vision-based approach for the direct measurement of displacements in vibrating systems," Smart Mater. Struct. Vol. 12, 2003, pp. 785-794.

[3] J. Lee, and M. Shinozuka, "A vision-based system for remote sensing of bridge displacement," NDT\&E International, Vol. 39, 2006, pp. 425-432.

[4] B. Pan, K. Qian, H. Xie, and A. Asundi, "Two-dimensional digital image correlation for in-plane displacement and strain measurement: a review," Vol. 20, 2009, pp. 062001.

[5] M. A. Sutton, J. L. Turner, H. A. Bruck, and T. A. Chao, "Full-field representation of discretely sampled surface deformation for displacement and train analysis", Experimental Mechanics, Vol. 31, 1991, pp. 168-177.

[6] S. Yoneyama, and Y. Morimoto, "Accurate displacement measurement by correlation of colored random patterns," JSME International Journal (Series A), Vol. 46, 2003, pp. 178-184.

[7] P. Ifju, B. Han, "Recent applications of moiré interferometry," Experimental Mechanics, Vol. 50, 2010, pp. 1129-1147.

[8] S. Kishimoto, Y. Tanaka, K. Naito, and Y. Kagawa, "Measurement of strain distribution of composite materials by electron moiré method," Nanocomposites with Unique Properties and Applications in Medicine and Industry, Dr. John Cuppoletti (Ed.), Chapter 11, InTech.

[9] Q. Wang, and S. Kishimoto, "Simultaneous analysis of residual stress and stress intensity factor in a resist after UV-nanoimprint lithography based on electron moiré fringes," J. Micromech. Microeng. Vol. 22, 2012, 105012 (7pp).

[10] S. Ri, S. Hayashi, S. Ogihara, and H. Tsuda "Accurate full-field optical displacement measurement technique using a digital camera and repeated patterns," Optics Express, Vol. 22, 2014, pp. 9693-9706.

[11] S. Ri, M. Fujigaki, and Y. Morimoto, "Sampling moiré method for accurate small deformation distribution measurement," Experimental Mechanics, Vol. 50, 2010, pp. 501-508.

[12] S. Ri, T. Muramatsu, M. Saka, K. Nanbara, and D. Kobayashi, "Accuracy of the sampling moiré method and its application to deflection measurement s of large-scale structures," Experimental Mechanics, Vol. 52, 2012, pp. 331-340.

[13] S. Ri, M. Saka, K. Nanbara, and D. Kobayashi, "Dynamic thermal deformation measurement of large-scale, high-temperature piping in thermal power plants utilizing the sampling moiré method and grating magnets," Experimental Mechanics, Vol. 53, 2013, pp. 1635-1646.

[14] W. B. Pennebaker, and J. L. Mitchellm, "JPEG Still Image Data Compression Standard,” New York, 1993. 